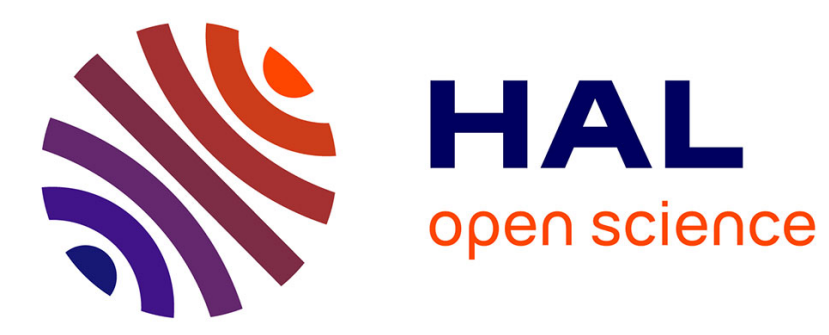

\title{
Analysis of the physical processes occurring during deep penetration laser welding under reduced pressure
}

Remy Fabbro, Koji Hirano, Shengyong Pang

\section{To cite this version:}

Remy Fabbro, Koji Hirano, Shengyong Pang. Analysis of the physical processes occurring during deep penetration laser welding under reduced pressure. Journal of Laser Applications, 2016, 28 (2), pp.022427. 10.2351/1.4944002 . hal-02292423

\section{HAL Id: hal-02292423 \\ https://hal.science/hal-02292423}

Submitted on 19 Sep 2019

HAL is a multi-disciplinary open access archive for the deposit and dissemination of scientific research documents, whether they are published or not. The documents may come from teaching and research institutions in France or abroad, or from public or private research centers.
L'archive ouverte pluridisciplinaire HAL, est destinée au dépôt et à la diffusion de documents scientifiques de niveau recherche, publiés ou non, émanant des établissements d'enseignement et de recherche français ou étrangers, des laboratoires publics ou privés. 


\title{
Analysis of the physical processes occurring during deep penetration laser welding under reduced pressure
}

\author{
Remy Fabbro \\ Laboratoire PIMM, Arts et Métiers ParisTech, 151 Bd. de l'Hôpital, Paris Cedex 75013, France \\ Koji Hirano \\ Nippon Steel and Sumitomo Metal Corporation, R\&D Planning Div., 20-1 Shintomi, Futtsu, Chiba 293-8511, \\ Japan \\ Shengyong Pang \\ State Key Laboratory of Material Processing and Die and Mould Technology, Huazhong University \\ of Science and Technology (HUST), Wuhan 430074, China
}

\begin{abstract}
Recent published experimental results obtained on deep penetration laser welding realized under reduced ambient pressure have shown very interesting results: resulting weld seams have geometrical characteristics that are similar to those obtained with electron beams. They show an increased penetration depth that can reach a factor two compared to atmospheric experiments, a larger aspect ratio with narrow and parallel sides of the weld seam. Also some humps around the rim of the keyhole appear. Of course, these modifications depend on the ambient pressure, but one also observes that these interesting improvements become independent of the ambient pressure below some critical pressure and also disappear at high welding speeds. Moreover, it is also observed that this critical pressure, below which these improvements do not vary, increases with the welding speed. In these previous publications, all these different characteristic results have not been explained. It is therefore the purpose of this paper to explain these different results. This has been obtained by using 3D numerical simulations of deep penetration laser welding and by studying the corresponding variation of physical parameters inside the keyhole (temperature, recoil pressure, and hydrodynamics of the vapor plume). An explanation for the evolution of these resulting weld seam geometries, as a function of the main operating parameters is proposed: ambient pressure, welding speed, and laser beam parameters (power and beam spot diameter). It is then possible to estimate this characteristic critical pressure, which is compared favorably with the corresponding previous experimental results. As a consequence, this analysis allows to define the optimum conditions for the improvement of the weld seam characteristics realized under reduced ambient pressure for an industrial environment. c○2016 Laser Institute of America.
\end{abstract}

Key words: laser welding, keyhole, reduced pressure, evaporation process

\section{INTRODUCTION}

Experiments of laser welding under reduced atmosphere have been realized since the early days of high power lasers. ${ }^{1,2}$ They all showed interesting properties for the resulting weld seams: a much deeper penetration depth, increased by as much as a factor of 2, with a better aspect ratio (depth over width). As these experiments were realized with a $\mathrm{CO}_{2}$ laser, the strong reduction of inverse bremsstrahlung (I. B.) effect was first mainly invoked for the explanation of this improvement. Indeed, accurate measurements of the evolution of the temperature and electron density inside the plasma plume for these reduced ambient pressures confirmed these points. ${ }^{3}$ Using local thermodynamic equilibrium of the plasma plume, it is easy to verify that the resulting electron density and therefore its temperature are decreased, when the ambient pressure is reduced. However, the reduction of vaporization temperature $T_{v}$ of the metals under reduced pressure was also a second argument for explaining these improvements: As $T_{v}$ decreases, less energy is necessary for generating the keyhole and so the incident power can be distributed along a greater depth.

Rather recent experiments have shown more complete and detailed results that confirm the previous one. ${ }^{4,5}$ But these experiments were realized using high power solid state lasers, so with a wavelength of typically $1.03-1.06 \mu \mathrm{m}$. It is known that I. B. absorption is scaling with the square of laser wavelength; so with a wavelength ten times shorter than with a $\mathrm{CO}_{2}$ laser, the I. B. absorption is 100 times less efficient, and therefore, this plasma shielding effect should not play a role anymore with solid state lasers.

In order to discuss more in detail the possible physical mechanisms that control the resulting keyhole behavior when laser welding is operated under reduced ambient pressures, the recent experimental results obtained by Abe et al. ${ }^{4}$ and Börner et al. ${ }^{5}$ have been used. These data are the only ones 
that give detailed quantitative data as a function of several operating parameters such as ambient pressure and welding speed, and therefore, they can be analyzed and discussed. As examples, Figs. 1 and 2 show characteristic variations of the resulting penetration depths, as a function of the ambient pressure, for different welding speeds and operating conditions. These results were obtained with a solid state laser.

Both set of results show a very similar behavior:

- Under low pressure conditions, the penetration depth improvement is only observed for rather low welding speeds. This improvement disappears typically above $6 \mathrm{~m} / \mathrm{min}$.

- Below some critical ambient pressure (in the 1-10 kPa range), which seems to slightly increase with the welding speed, there is no variation of the penetration depth.

According to literature review, no explanation has been given for these very characteristic similar experimental results. It is therefore the aim of this paper to discuss the possible mechanisms controlling this behavior that will be detailed in the following sections II-A and B. One can also add that other experimental observations were obtained during these experiments. For example, a strong reduction of the luminosity of the vapor plume was observed under vacuum conditions, which is likely correlated to the decrease of its density. Also, the keyhole aperture becomes enlarged, and the melt pool is much more stable.

The paper is constructed as follows: in the first part of Sec. II, several mechanisms that have been already invoked for the explanation of the penetration improvement under vacuum conditions when using solid state lasers are discussed. Then recent 3D numerical simulations for analyzing the effect of welding speed on penetration depth variation will be used. Finally in the last part, the observed saturation of the penetration depth below a critical pressure will be explained.

\section{INVOLVED MECHANISMS WHEN USING SOLID STATE LASER}

As previously discussed in the Introduction, when using a laser wavelength of about $1 \mu \mathrm{m}$, delivered by a solid state

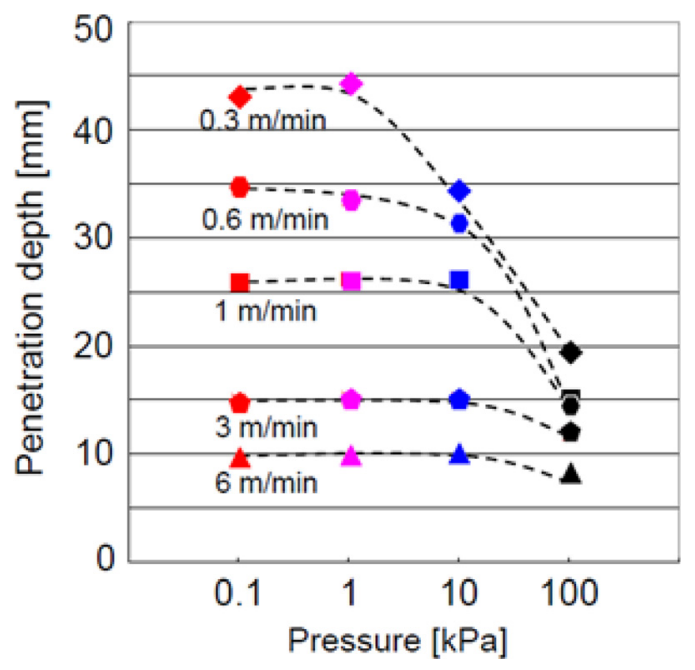

FIG. 1. Penetration depths as a function of ambient pressure for different welding speeds from Ref. 4. 304 stainless steel; laser power: $16 \mathrm{~kW}$; focal spot diameter: $0.4 \mathrm{~mm}$.

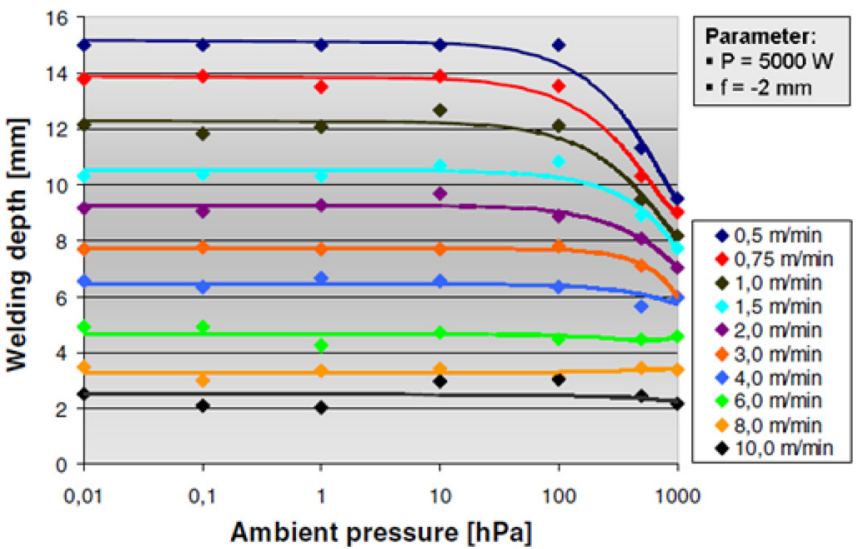

FIG. 2. Penetration depths as a function of ambient pressure for different welding speeds from Ref. 5 . Mild steel; laser power: $5 \mathrm{~kW}$; focal spot diameter: $0.3 \mathrm{~mm}$.

laser, the I. B. absorption inside the vapor plume is completely negligible. These conditions result from a feedback mechanism of the low ionization of the vapor plume due to its low temperature (which is typically the evaporation temperature of the material) controlled by the low I. B. absorption. Therefore, the improvement of the penetration depth observed for reduced ambient pressure cannot result from the decrease of the electron density with the ambient pressure.

\section{A. Effect of laser beam scattering processes}

It is known that when the laser wavelength is decreased, some other mechanisms, such as Rayleigh or Mie scattering, begin to play a role for the absorption and the scattering of the incident laser beam, along its propagation through the vapor plume on particles present in the vapor plume. ${ }^{6-8}$ These particles result from coalescence of metallic atoms due to the condensation process occurring inside the vapor plume. This scattering occurs on small particles with average diameters around $100 \mathrm{~nm}$ that depend on the shielding gas used, ${ }^{7,8}$ and these particles can cluster together by condensation during the vapor plume expansion. ${ }^{9}$ As the Rayleigh scattering loss is inversely proportional to the fourth power of the wavelength, therefore, this scattering effect is much more dominant at short laser wavelength. However, Mie scattering becomes important when the radius of the particles is of the order of magnitude of the laser wavelength. Despite the detailed knowledge of the Rayleigh and Mie scattering coefficients, a precise estimation of the effect on beam absorption and diffusion is very difficult because the volume ratio occupied by these particles inside the vapor plume is not known and rough estimations must be used. Many experimental data using transverse probe beam of the vapor plume have given a lot of dispersion for these absorption data. ${ }^{6-8,10}$ However, the very recent work of Shcheglov ${ }^{8}$ seems to have brought the deepest analysis of these different mechanisms of plume scattering. He concluded that the total attenuation of the laser beam propagating through the vapor plume to the sample surface was estimated to be about $10 \%$ for high power fiber laser welding of mild steel. Moreover, even if this beam attenuation is not so high, taking into account the 
temporal response of the evaporation process inside the keyhole, he also concluded that this perturbation of the beam propagation should have a negative impact on the stability of the laser beam entering the keyhole and as a result, on the melt pool dynamics.

If the welding is realized under vacuum conditions, it is expected that the vapor plume density above the keyhole is strongly decreased (see below Eq. (8)), and correspondingly so is the particle density. One can thus expect that the aforementioned beam absorption is quite suppressed. But it is clear that this increase of $10 \%-20 \%$ of the incident laser power entering the keyhole alone cannot produce the observed improvement by quite a factor of 2 of the penetration depth. Other options taking into account the state of the vapor inside the keyhole must be considered. Unfortunately, there are no studies of the state of the vapor inside the keyhole, and mechanisms of particle generation for these conditions are not clear at all and should need specific detailed investigations. ${ }^{11}$ If microdroplets or aggregates are present inside the keyhole, their density should be much higher than in the plume, outside the keyhole (where the plume undergoes an expansion). So one can expect that the previous scattering processes could be much more important inside the keyhole and consequently, when the ambient pressure is decreased, their effect could be strongly reduced.

One can add that even if the above-mentioned level of absorption by the plume (about 10\%-20\%) does not explain the observed penetration improvement, it is known that in case of atmospheric experiments, the suppression of these perturbing effects by using a transverse cross jet that blows the vapor plume or coaxial shielding jet that crushes the vapor plume, improves the weld pool stability and gives similar improvement of stability as those observed by Abe et $a l .{ }^{4}$ under vacuum conditions.

\section{B. Effect of the decrease of evaporation temperature}

So the last mechanism that was proposed for explaining the strong improvement of penetration depth concerns the reduction of the evaporation temperature of metals under reduced ambient pressure. ${ }^{1,2,12,13}$ For the sake of simplicity, one usually uses the Clausius-Clapeyron relation for defining the metallic vapor pressure $P_{\mathrm{CC}}\left(T_{\mathrm{s}}\right)$ in equilibrium with the liquid metal at a temperature $T_{s}$. This well-known relation $P_{\mathrm{CC}}\left(T_{s}\right)$ is given by

$$
P_{\mathrm{CC}}\left(T_{S}\right)=P_{0} \exp ^{\left(B\left(1-\left(T_{V} / T_{S}\right)\right)\right)},
$$

where $P_{0}(=1 \mathrm{bar})$ is the vapor pressure corresponding to the evaporation temperature $T_{v}$ at standard atmosphere (1 bar). In Eq. (1), $B=L_{\text {vap }} A / R T_{v}$, where $L_{\text {vap }}$ is the latent heat of evaporation $(\mathrm{J} / \mathrm{kg}), A$ is its molecular weight $(\mathrm{kg})$, and $R=8.32 \mathrm{~J} / \mathrm{K}$ (one can verify that for most of used metals, $12 \leq B \leq 14)$.

Using Eq. (1), it is easy to see that for iron (for which $B=13.57$ ), the evaporation temperature is decreased by $800 \mathrm{~K}$, when the vapor pressure is varied from 100 to $1 \mathrm{kPa}$. One can estimate the improvement resulting of this decrease of evaporation temperature on the penetration depth. By using the $2 \mathrm{D}$ heat flow solutions, ${ }^{14,15}$ it is possible to determine the power per unit depth $q$ (in $\mathrm{W} / \mathrm{m}$ ) necessary for moving a cylinder with a radius $r$ and a surface temperature $T_{v}$, at a welding speed $V$ inside a material whose initial temperature is $T_{0}$. This solution, derived at low welding speed (because it is the regime where the penetration depth improvement is experimentally observed), is given by

$$
q=\frac{P}{L_{\mathrm{KH}}} \approx \frac{6.3 K\left(T_{V}-T_{0}\right)}{\operatorname{Ln}\left(\frac{2.25 \kappa}{V \cdot r}\right)} .
$$

In the above equation, $P$ is the incident laser power absorbed along the keyhole of length $L_{\mathrm{KH}}$. $K$ and $\kappa$ are, respectively, the heat conductivity and diffusivity of the material.

Let us consider the welding of iron, at a welding speed $V=2 \mathrm{~m} / \mathrm{min}$ and with a keyhole radius $r=0.25 \mathrm{~mm}$ : the power per unit depth necessary for maintaining this keyhole decreases from 0.77 to $0.55 \mathrm{~kW} / \mathrm{mm}$ when the evaporation temperature decreases by $800 \mathrm{~K}$. Therefore, as the same laser power is distributed on a greater depth $L_{\mathrm{KH}}$, this corresponds to a $40 \%$ increase of the penetration depth.

Using these simplified models, one can see that the combined contribution of these two mechanisms, reductions of the evaporation temperature and the scattering by the plume, and possibly inside the keyhole too, could quite explain the observed improvement of about $100 \%$ of the keyhole depth under vacuum conditions.

Now, in order to have deeper insights into some mechanisms, and particularly those related to the effect of welding speed, recent results of 3D nonstationary hydrodynamic numerical simulations of the laser welding process are recalled here. ${ }^{16}$ Without entering in the description of the detailed physics used in these simulations, one can recall that Navier-Stokes equations were used to determine the fluid flow inside the melt pool, with its free surface dynamics controlled by surface tension and the Marangoni effect. Also, inside the keyhole, multiple beam reflexions, with Fresnel local absorptivity were taken into account in order to estimate the absorbed intensity. The driving mechanism for keyhole generation is the vapor flow due to evaporation that generates a recoil pressure. In order to simplify these simulations, this vapor flow was not described, but its effect was considered by using a recoil pressure $P_{r}\left(T_{s}\right)$ that depends on the local surface temperature $T_{s}$. The starting point for its description uses the previous Clausius-Clapeyron Eq. (1) only valid for equilibrium conditions, which is modified by taking into account the incomplete recondensation process ${ }^{17,18}$

$$
P_{r}\left(T_{S}\right)=\left(\frac{1+\beta}{2}\right) P_{\mathrm{CC}}\left(T_{S}\right)
$$

where $\beta$ is the condensation coefficient (fraction of recondensation particles to evaporations ones), which depends on the Mach number at the exit of the Knudsen layer, whose value is controlled by the boundary limits of the vapor flow. It is known that $\beta$ decreases from 1 (at equilibrium, for low evaporation rates or high ambient pressures) to 0.18 for high evaporation rates (under vacuum conditions, at high temperature, or high laser intensity). ${ }^{17-19}$ 
However, in case of an ambient atmosphere, this relation was modified by taking into account the experimental observation $^{20}$ that the ambient atmosphere confines the metallic vapor when the temperature of the liquid surface is below or around the evaporation temperature $T_{v}$ (corresponding to the ambient pressure). In this case of "diffusive" evaporation, there is no surface depression and $\beta=1$. For surface temperatures exceeding this evaporation temperature, the evaporation rate and surface depression strongly increase, and limiting values of $\beta=0.18$ can be reached. So, it is important to recall the global evolution of the recoil pressure: below $T_{v}$, the pressure exerted on the surface is $P_{\text {amb; }}$; above $T_{v}$, there is a transition, where the recoil pressure increases and reaches at high surface temperature, the limit given in Eq. (3), with $\beta=0.18$. There is currently no theoretical model that describes this transition curve because the condensation coefficient $\beta$ depends on the characteristics of the global vapor flow, and more precisely, of the Mach number at the exit of the Knudsen layer.

Figure 3 resumes these evolutions of the recoil pressure $P_{r}\left(T_{s}\right)$ as a function of the surface temperature $T_{s}$, for two different ambient pressures. The reduction of evaporation temperature is of course also evidenced in this description.

Figure 4 recalls some examples of simulation results that were obtained for two different ambient pressures: at atmospheric pressure and under complete vacuum. ${ }^{16}$ One can see that the penetration depth increases from 1.5 to $2.5 \mathrm{~mm}$, and the cross section of the weld seam is also modified with a greater aspect ratio under vacuum conditions. The analysis of the simulations shows that the melt flow is strongly modified by the reduction of keyhole surface temperature: for atmospheric ambient pressure, the high temperature reduces the keyhole depth and enlarges the melt pool by heat diffusion allowing complex 3D flow that results in the usual "wine cup shape."16

The variation of the average keyhole front temperature for these two conditions is reported in Fig. 5, as a function of the welding speed.

Of course, the keyhole temperature at low ambient pressure is smaller, for the reasons discussed previously. One can

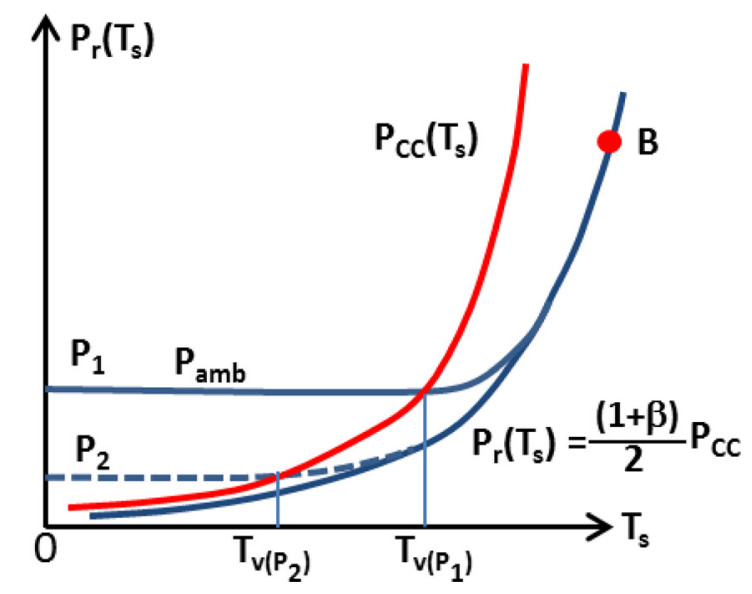

FIG. 3. For two different ambient pressures $P_{\mathrm{amb}}, P_{1}$ and $P_{2}$, examples of evolution of the recoil pressure $P_{r}\left(T_{s}\right)$ as a function of surface temperature $T_{s} . T_{v}$ is the evaporation temperature such as $P_{\mathrm{CC}}\left(T_{v}\right)=P_{\mathrm{amb}}$. For $T_{s}>T_{v}, \beta$ decreases from 1 to 0.18 .
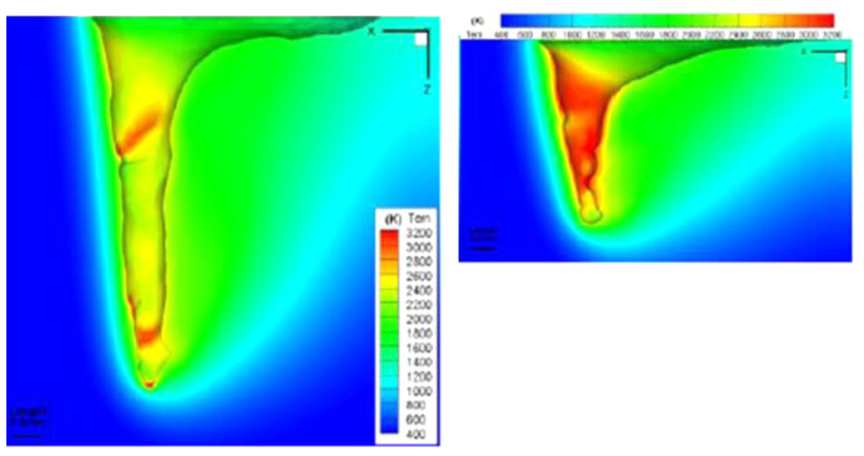

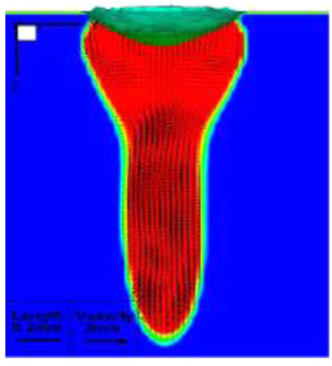

$\mathrm{P}_{\mathrm{amb}}=0 \mathrm{kPa}$

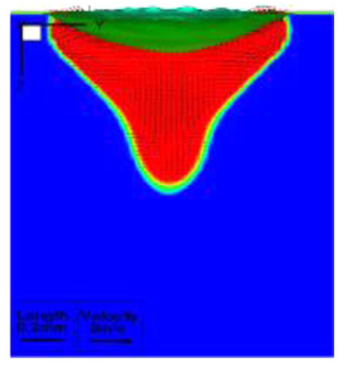

$\mathrm{P}_{\mathrm{amb}}=100 \mathrm{kPa}$
FIG. 4. Example of longitudinal and transverse views of the melt pool obtained under vacuum and atmospheric pressure (Ref. 16).

also observe that these temperatures increase with the welding speed and the slope of this increase is greater for low pressure experiments. This can be explained if one considers a same increase $\Delta V$ of the welding speed at both pressures: this increase of $\Delta V$ induces an increase of the dynamic pressure of the melt flow incoming through the keyhole front, which must be equilibrated by a corresponding increase of the recoil pressure $\Delta P_{r}$, and so an increase $\Delta T$ of the surface temperature following Eq. (3) is necessary. Because of the exponential dependence of recoil pressure with temperature, this increase $\Delta T$ of temperature will be greater at low ambient pressure than at atmospheric pressure.

Now, as the temperature increases with the welding speed, if the welding speed is high enough, the resulting temperature (and the corresponding recoil pressure) will be

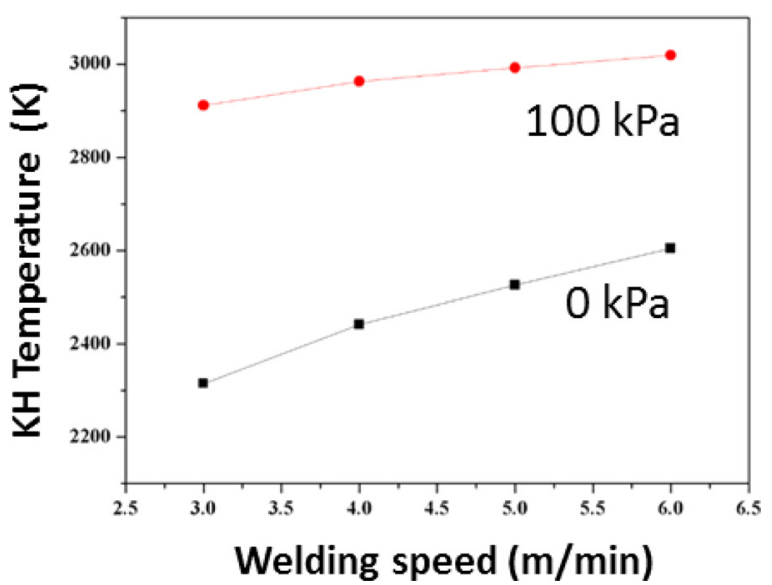

FIG. 5. Mean keyhole front temperature dependence with the welding speed, under atmospheric pressure and vacuum. (Laser power: $2 \mathrm{~kW}$, welding speed: $3 \mathrm{~m} / \mathrm{min}$; beam radius: $0.25 \mathrm{~mm}$; 304SS) (Ref. 16). 
much greater than the boiling temperature $T_{v}$ or the ambient pressure. In the $\left(P_{r}\left(T_{s}\right), T_{s}\right)$ diagram (see Fig. 3), if this corresponding state is for example located at point $B$, which lies above the transition curve from $P_{\mathrm{amb}}$ to $0.5(1+\beta) P_{\mathrm{CC}}\left(T_{s}\right)$, any reduction of $P_{\mathrm{amb}}$ should not affect the coordinates of this point $B$, i.e., the average keyhole temperature should be quite the same. So, for high welding speeds, the average keyhole temperature and therefore the penetration depth do not depend on the ambient pressure, in agreement with the experimental observations.

\section{ANALYSIS OF THE OBSERVED SATURATION OF THE PENETRATION DEPTH WITH DECREASING AMBIENT PRESSURE}

One of the striking results of the previously discussed experiments (see Figs. 1 and 2) is the saturation of the penetration depth below some critical ambient pressure. It can also be observed that this critical pressure seems to increase with the welding speed. This experimental result can be understood if one analyses the evolution of the total pressure $P_{0}$ that must exist inside the keyhole in order to maintain it opened. This total pressure $P_{0}$ can be written as

$$
P_{0}=P_{\mathrm{amb}}+P_{c}+P_{h}+P(V) .
$$

Let us discuss the different terms in Eq. (4):

- $P_{\mathrm{amb}}$ is the ambient pressure transmitted through the liquid melt pool.

- $P_{c}=\sigma / r$ is the closing pressure of the keyhole defined by the surface tension $\sigma$ of the liquid and its radius $r$ (which is of the order of magnitude of the focal spot radius).

- $P_{h}=\rho_{1} g L_{\mathrm{KH}}$ is the maximum hydrostatic pressure resulting from a liquid thickness corresponding to the keyhole depth ( $\rho_{1}$ : liquid density, $g=9.71 \mathrm{~m} \mathrm{~s}^{-2}$ is the gravity; $L_{\mathrm{KH}}$ is the keyhole depth). Of course, the hydrostatic pressure varies from 0 at the keyhole surface to $P_{h}$ at its bottom.

- $P(V)$ is a function that takes into account the displacement of the keyhole with a welding speed $V$ and of course increases with it.

As these experiments are realized at low welding speed, one can neglect in a first approach $P(V)$. Also, for usual values of the different parameters involved in Eq. (4), one can also neglect $P_{h}\left(P_{h} \approx 1.6 \mathrm{kPa}\right.$ for $\left.L_{\mathrm{KH}}=20 \mathrm{~mm}\right)$, compared to $P_{c} \approx 10 \mathrm{kPa}$, (with $\sigma \approx 2 \mathrm{~N} / \mathrm{m}$ and $r \approx 0.2 \mathrm{~mm}$ ). So Eq. (4) becomes

$$
P_{0} \approx P_{\mathrm{amb}}+P_{c} .
$$

From the above equation, it is clear that the minimum pressure inside the keyhole is its closing pressure $P_{c}(=\sigma / r)$, which is obtained under complete vacuum. Hence, the lower limit of evaporation temperature inside the keyhole will be defined by this pressure $P_{c}$. So one can define a critical ambient pressure

$$
P_{\mathrm{CR}}=P_{c} / 10
$$

below which the effect of ambient pressure can be considered negligible in Eq. (5). Typically, $P_{\mathrm{CR}}$ is about $1 \mathrm{kPa}$.
If one considers the results of Abe et al. (using $r \approx 0.2 \mathrm{~mm}, \sigma \approx 2 \mathrm{~N} / \mathrm{m})$, one finds that $P_{\mathrm{CR}} \approx 1 \mathrm{kPa}$ and experimentally, the saturation is observed below $1-2 \mathrm{kPa}$. For the results of Börner et al. ( $r \approx 0.15 \mathrm{~mm}$, and $\sigma \approx 2 \mathrm{~N} / \mathrm{m})$, $P_{\mathrm{CR}} \approx 1.2 \mathrm{kPa}$ and experimentally the saturation is also observed below $\approx 1 \mathrm{kPa}$. So, one can consider that a rather good agreement is achieved with these published experimental data.

Remark 1. If the welding speed increases, the term $P(V)$ should be also taken into account into Eqs. (5) and (6) and it increases with $V$. Therefore, the critical pressure $P_{\mathrm{CR}}$ should also slightly increase with the welding speed $V$. Even if it is not striking, this effect can also be observed on the experimental results in Figs. 1 and 2.

Remark 2. The reduction of the ambient pressure on the ejection velocity of the vapor plume and on the resulting induced upward melt flow driven by its friction on the keyhole wall can also be studied. Let us consider that Bernoulli equation can be used in order to estimate the ejection velocity $V_{\text {eject }}$ of the vapor plume. (This assumption is valid if the exit Mach number of the flow is small.) The vapor is accelerated from 0 (inside the keyhole, at a pressure $P_{0}$ ) to $V_{\text {eject }}$ (outside the keyhole, at a pressure $P_{\mathrm{amb}}$ ) by this difference of pressure. One then can write

$$
P_{0}-P_{\mathrm{amb}}=0.5 \rho_{g} V_{\text {eject }}^{2}=\sigma / r
$$

where $\rho_{g}$ is the vapor density that can be obtained if one considers that the vapor plume follows the equation of state of a perfect gas, at evaporation temperature $T_{v}$. The vapor plume pressure is in equilibrium with the ambient pressure

$$
P_{\mathrm{amb}}=\rho_{g} R T_{v} / A .
$$

Combining Eqs. (7) and (8), and introducing the sound speed inside the vapor $C_{s}=\left(\gamma R T_{v} / A\right)^{1 / 2}(\gamma$ is the specific heat capacity ratio), one obtains

$$
V_{\text {eject }}=\left(\frac{2 R T_{V} P_{C}}{A P_{\mathrm{amb}}}\right)^{1 / 2},
$$

and the Mach number at the keyhole exit

$$
M=\frac{V_{\text {eject }}}{C_{S}}=\left(\frac{2 P_{c}}{\gamma P_{\mathrm{amb}}}\right)^{0.5} .
$$

For example, with $P_{\mathrm{amb}}=100 \mathrm{kPa}$ and $P_{c}=10 \mathrm{kPa}$, for a vapor plume of iron $(\gamma=5 / 3$ for a monoatomic gas $)$, $V_{\text {eject }} \approx 300 \mathrm{~m} / \mathrm{s}$ and $M \approx 0.3$. This value of $M \approx 0.3$ is the limit acceptable for using Bernoulli equation (7) for a compressible fluid.

The upward melt flow velocity $U$ induced by the vapor ejection inside the keyhole can be also estimated by considering that the shear stress of the vapor $\tau_{v}$ at the keyhole wall is equal to the shear stress $\tau_{1}$ inside the liquid $^{21}$

$$
\tau_{v}=\frac{4 n_{v} V_{\text {eject }}}{r}=\tau_{1}=n_{1} \frac{U}{\delta},
$$

where $\eta_{v}$ and $\eta_{1}$ are the viscosity of the vapor plume (that does not depend on its density), and the viscosity of the melt 
pool respectively. $\delta\left(\approx 5 L_{\mathrm{KH}} \mathrm{Re}^{-0.5}\right.$ ) is the boundary layer developed inside the liquid melt pool (that depends on its Reynolds number Re). The final scaling law for the upward melt flow velocity $U$ is then

$$
U \approx V_{\text {eject }}^{1 / 3} L_{\mathrm{KH}}^{1 / 3} \approx\left(\frac{L_{\mathrm{KH}}}{P_{\mathrm{amb}}}\right)^{1 / 3} .
$$

Experimentally, it is observed that when the ambient pressure $P_{\mathrm{amb}}$ is decreased by a factor of 10 , the keyhole depth is increased by a factor of about 2. Using Eq. (12), one can see that the upward melt flow velocity $U$ could be increased by about $2.5, U$ varying from about 4 to $10 \mathrm{~m} / \mathrm{s}$. Therefore, one can think that this increase of $U$ for low ambient pressures is the reason of the appearance of large bulges of melt around the rim of the keyhole exit, for these conditions. ${ }^{22}$

Remark 3. It is interesting to notice that a keyhole can be considered as a nozzle that ejects a vapor inside an ambient atmosphere and one knows that a threshold condition for observing a sonic-supersonic transition at the exit of this nozzle is given by the relation ${ }^{23}$

$$
\frac{P_{0}}{P_{\mathrm{amb}}}=K=\left(\frac{\gamma+1}{2}\right)^{(\gamma /(\gamma-1))} .
$$

With $\gamma=5 / 3, K=2.05$. As $P_{0}=P_{\mathrm{amb}}+P_{c}$, it easy to see that as soon as the condition

$$
P_{\mathrm{amb}} \leq P_{c}
$$

is verified, there should be a shock wave at the exit of the keyhole and the vapor flow is then choked. One can see that this condition is fulfilled when the ambient pressure is lower than the critical one (Eq. (6)), i.e., when the penetration depth saturation previously discussed occurs. The experimental verification of the presence of this shock wave at the keyhole exit, for these pressure conditions, would be an interesting confirmation of the validity of this complete model. However, because of the involved low pressures, usual techniques of optical strioscopy that are used, for example, for similar visualizations during percussion laser drilling experiments achieved under atmospheric pressure ${ }^{24}$ may not be sensitive enough.

\section{CONCLUSION}

This paper has analyzed the different physical mechanisms that are the origins of the observed improvements during solid state laser welding under reduced pressure. The conclusions are:

- The improvement by quite a factor two of the penetration depth results mainly from the reduction of the evaporation temperature under low ambient pressure. As the evaporation needs a lower temperature, less power per unit depth of keyhole is necessary, therefore, the incident laser power can be distributed along a greater depth.

- The reduction with the ambient pressure of the scattering processes by the particles or aggregates presenting inside the vapor plume, and possibly inside the keyhole, which are favored by the use of short laser wavelength, is also another probable mechanism. However, further investigations are needed in order to have decisive conclusions.

- The saturation of the penetration depth below some critical pressure is another important effect of the reduction of the ambient pressure, which is observable only at low welding speeds. A modification of the recoil pressure law has been proposed for the interpretation of these different results.

- At high welding speeds, the recoil pressure is much higher than the ambient one, so any reduction of the ambient pressure cannot modify the evaporation temperature inside the keyhole.

- At low welding speeds, the pressure inside the keyhole decreases with the ambient one and is limited by the closing pressure $P_{c}=\sigma / r$, necessary for maintaining the keyhole opened. Therefore, this closing pressure will define the minimum evaporation temperature occurring inside a keyhole. A critical pressure $P_{\mathrm{CR}} \approx 0.1 P_{c}$ below which the saturation of the penetration depth occurs has been defined, and also the small increase of this critical pressure with the welding speed has been explained. One must point out the importance in these explanations of the role played by the surface tension $\sigma$ of the used material, and more particularly by the beam radius $r$, which could be easily modified in future similar experiments in order to confirm this interpretation for a more extended range of parameters.

Beyond the interpretation of these different mechanisms, one interest of this paper is to show that for industrial applications of laser welding under reduced pressure, which begin to be used (in the gear manufacture of automobile production, for example) due to these several advantages, it is not necessary to have a very good level of vacuum, as it is the case for the usual electron-beam welding. It should be useful in the design and in the simplification of the corresponding industrial vacuum chambers.

${ }^{1}$ Y. Arata, N. Abe, T. Oda, and N. Tsujii, "Fundamental phenomena during vacuum laser welding," in Proceedings of the ICALEO, LIA Boston, MA, USA, 1985, Vol. 44, pp. 1-7.

${ }^{2}$ C. O. Brown and C. M. Banas, "Laser welding in reduced pressure atmospheres," in Proceedings of the ICALE0-83, Los Angeles, CA, USA, 1983.

${ }^{3}$ A. Verwaerde, R. Fabbro, and G. Deshors, "Experimental study of continuous $\mathrm{CO}_{2}$ laser welding at subatmospheric pressures," J. Appl. Phys. 78, 2981-2984 (1995).

${ }^{4}$ Y. Abe, M. Mizutani, Y. Kawahito, and S. Katayama, "Deep penetration welding with high power laser under vacuum," in Proceedings of the ICALEO 2010, LIA, Anaheim, CA, USA, 2010, pp. 648-653.

${ }^{5}$ C. Börner, K. Dilger, V. Rominger, T. Harrer, T. Krussel, and T. Lower, "Influence of ambient pressure on spattering and weld seam quality in laser beam welding with the solid-state laser," in Proceedings of the ICALEO 2011, LIA, Orlando, FL, USA, 2011, pp. 621-629.

${ }^{6} \mathrm{D}$. Lacroix, G. Jeandel, and C. Boudot, "Solution of the radiative transfer equation in an absorbing and scattering Nd:YAG laser-induced plume," J. Appl. Phys. 84, 2443-2449 (1998).

${ }^{7}$ J. Greses, P. A. Hilton, C. Y. Barlow, and W. M. Steen, "Plume attenuation under high power Nd:yttritium-aluminum-garnet laser welding," J. Laser Appl. 16, 9-15 (2004). 
${ }^{8}$ P. Shcheglov, Study of Vapour-Plasma Plume During High Power Fiber Laser Beam Influence on Metals, BAM-Dissertationsreihe - Band 88, Berlin, Germany, 2012.

${ }^{9}$ A. Matsunawa, S. Katayama, S. Susuki, and T. Ariyasu, "Laser production of metallic ultrafine particles," Trans. JWRI 15(2), 61-72 (1986).

${ }^{10}$ S. Katayama, A. Yohei, M. Mizutani, and Y. Kawahito, "Development of deep penetration welding technology with high brightness laser under vacuum," Phys. Procedia 12, 75-80 (2011).

${ }^{11} \mathrm{~S}$. Kotake, "Formation of metal and non-metal clusters through laser and electron beam vaporisation," in Heat and Mass Transfer in Materials Processing, edited by I. Tanasawa and N. Lior (Hemisphere Publishing Corporation, 1992), p. 107.

${ }^{12}$ M. Kabasawa, M. Ono, K. Nakada, and S. Kosuge, in Laser Treatment of Materials, edited by B. L. Mordike (DGM-Informationsgesellschaft, Verlag, 1992), p. 667.

${ }^{13}$ C. Banas, "High power laser welding," in The Industrial Laser Annual Handbook, edited by D. Belforte and M. Levitt (PennWell Books, Tulsa, OK, 1986), pp. 69-85.

${ }^{14}$ H. C. Carslaw and J. C. Jaegar, Conduction of Heat in Solids (Clarendon Press, Oxford, 1959).

${ }^{15}$ D. T. Swift-Hook and A. E. F. Gick, "Penetration welding with lasers," Weld. Res. Journal 52(11), 492s-499s (1973).

${ }^{16}$ S. Pang, K. Hirano, R. Fabbro, and T. Jiang, "Explanation of penetration depth variation during laser welding under variable ambient pressure," J. Laser Appl. 27, 022007 (2015).

${ }^{17}$ C. J. Knight, "Theoretical modeling of rapid surface vaporization with back pressure," AIAA J. 17, 519-523 (1979).

${ }^{18}$ A. A. Samokhin, "First-order phase transitions induced by laser radiation in absorbing condensed matter," in Proceedings of the Institute of General Physics: Effect of Laser Radiation on Absorbing Condensed Matter, edited by A. M. Prokhorov (Nova Science Publishers, Commack, NY, 1990), pp. $1-161$.

${ }^{19}$ S. I. Anisimov, "Vaporization of metal absorbing laser radiation," Sov. JETP 27(1), 182-183 (1968).

${ }^{20}$ K. Hirano, R. Fabbro, and M. Muller, "Experimental determination of temperature threshold for melt surface deformation during laser interaction on iron at atmospheric pressure," J. Phys. D: Appl. Phys. 44, 435402 (2011).
${ }^{21}$ R. Fabbro, M. Hamadou, and F. Coste, "Metallic vapor ejection effect on melt pool dynamics in deep penetration laser welding," J. Laser Appl. 16, 16-19 (2004).

${ }^{22}$ U. Reisgen, S. Olschok, and S. Jakobs, "Laser beam welding under vacuum of a fine-graded steel," in Proceedings of the ICALEO'2014 Conference (LIA), San Diego, USA, 2014, pp. 933-939.

${ }^{23}$ M. C. Potter and D. C. Wiggert, Mechanics of Fluids, 3rd ed. (Cengage, 2010).

${ }^{24}$ M. Schneider, L. Berthe, R. Fabbro, M. Muller, and M. Nivard, "Gas investigation for laser drilling," J. Laser Appl. 19(3), 165-169 (2007).

\section{Meet the Authors}

Remy Fabbro is presently Emeritus Research Director in CNRS (French National Civil Research Center). He has always been concerned in the study of laser-matter interaction and since 1989, in the fundamental processes related to different industrial laser applications such as laser welding, cutting, drilling, and shock processing by using both experimental and modeling approaches.

Koji Hirano is a senior manager of R \& D Planning Div., Nippon Steel \& Sumitomo Metal Corporation. He joined the company in 2003 and worked on R \& D of laser metal processing until 2014. He was engaged in Ph.D. study at Arts et Métiers ParisTech from 2009 to 2011 and obtained Ph.D. in 2012.

Shengyong Pang is an Assistant Professor of Huazhong University of Science and Technology (HUST), China. He received his Bachelor, Master, and Ph.D. degrees from HUST in 2004, 2006, and 2011, respectively. His research interests include laser material processing, welding, and 3D printing. 